Mike Halling

Aug. 13, 1991

PBAR Note $x x x$

Debuncher Cooling Limitations to stacking

\title{
INTRODUCTION
}

During the January studies period we performed studies to determine the effect that debuncher cooling has on the stacking rate. Two different sets of measurements were made separated by about a week. Most measurements reported here are in PBAR $\log 16$, page 243-247. These measurements were made by changing the accelerator timeline to give about 6 seconds between 29 's, and then gating the cooling systems to simulate reduced cycle times. For the measurement of the momentum cooling effectiveness the gating switches could not be made to work, so the timeline was changed for each measurement. The cooling power of all three systems was about 800 watts for the tests reported here. We now regularly run at 1200 watts per system.

\section{DESCRIPTION DF MEASURED QZUANTITIES}

Any test of the stacking efficiency must in the end run be made using A:PRDCTN. This measurement is based on beam toroids and the DCCT, and is therefore relatively free of long term systematic errors. The disadvantage is that it takes a long time to make a good statistical measurement, roughly about 10 minutes for a precision of about 5\%, and this anti-proton collection efficiency includes many different sources of large losses that vary by about $10 \%$ or more from hour to hour. For these and other reasons it is often best to look at more specific measurements of efficiency. The machine must run smoothly for the duration of the measurement, which often is not possible.

The bunch rotation efficiency, D:FFTEFF, is normally measured by the FFT a few $1 / 10$ 's of a second after injection. It is the ratio of antiproton current within momentum spread $.2 \% \mathrm{dp} / \mathrm{p}$ to the total antiproton current. The time that this measurement is made can be varied to show the effects of momentum cooling. 
The effect of transverse cooling can be seen most directly by looking at the D->A line SEM's. Changing the cooling time changes the width on SEM806 and SEM807.

The D->A efficiency is measured by the ratio

A:FFTTOT/D:FFTTOT. Since the circumference of the two machines are not the same this ratio should be 1.06 for $100 \%$ transmission.

A:FFTEFF is the ratio of beam within $.2 \% \mathrm{dp} / \mathrm{p}$ in the accumulator to the beam within . $2 \% \mathrm{dp} / \mathrm{p}$ in the debuncher. Without momentum cooling this would be a good measurement of the $D->A$ transfer efficiency for particles within the momentum aperture of the accumulator, however, the debuncher measurement is made before the beam has been cooled and the accumulator measurement is made after cooling. A:FFTEFF can often exceed 150\%, which simply shows that momentum cooling is working.

By changing the trigger time for the FFT it is possible to measure the amount of beam left behind by ARF1. A simple way of doing this is to measure A:FFTTOT/D:FFTTOT with D:FFT1=1200000.

The overall efficiency of transporting beam from the injection orbit to the core can be measured by plotting A:STCKRT/A:FFTTOT. To convert this to an efficiency one needs to multiply by $10 * * 6 * 120 /(3600 * 19)$; which is the correction factor for 19 pulses/supercycle with a 120 second supercycle. This efficiency includes the beam left on the injection orbit by ARF1, so for many of plots below the ARF1 loss is added back in.

\section{RESULTS}

The measurements were taken with an accelerator timeline that had 19 pulses per 120 second supercycle, resulting in 6 seconds between pulses. Gating switches were used on the horizontal and vertical cooling to limit their cooling time. The momentum cooling and stack tail cooling were left on for the entire time.

Figure 1 shows the bunch rotation efficiency measured at different times in the cycle. There is a very clear increase with time, showing that momentum cooling is indeed working. It looks like 2 seconds of momentum cooling at 800 watts is not enough to 
push all the beam into the center, but it is enough time to make significant improvement. After 5 seconds of cooling $90 \%$ of the beam is within the $.2 \% \mathrm{dp} / \mathrm{p}$ window.

Figure 2 shows the net antiproton production efficiency as a function of the transverse cooling time. With 6 seconds of cooling the production efficiency was about 13 . When the transverse cooling is limited to 2 seconds the efficiency is reduced to about 9. The increase in efficiency due to transverse cooling has two components, shown in Figures 3 and 4 . The D->A transfer efficiency is shown in Figure 3. With 6 seconds of cooling this approaches 100\%. Notice that A:FFTEFF is 150\% with 6 seconds of cooling. In addition, the transfer efficiency from the accumulator injection orbit to the core also has a slight increase with increased cooling time as is shown in Figure 4. Perhaps some of the beam is lost in the stack tail due to aperture restrictions on the way into the core.

Figure 5 shows a measurement of the ARF1 capture efficiency. The loss of about $15 \%$ of the beam was invariant with cooling time. Figure 6 shows the net production efficiency as a function of momentum cooling time, holding the transverse cooling at 2 seconds. This measurement was made by changing the accelerator timeline for each point. In this plot $I$ have added a point for no momentum cooling taken during the second week of studies. The measurement at 2.4 seconds appears to be anonymously low. It is clear that momentum cooling improves the stacking rate, it is not clear wether 2.4 seconds of momentum cooling is enough at 800 watts.

Figures 7 and 8 show the effect of transverse cooling on the beam size in the $D->A$ channel. It is clear that increasing the cooling time reduces the emmitance of the beam in the debuncher.

\section{CONCLUSION}

All three cooling systems are necessary for the best stacking rates. None are saturated, the stacking rate would improve if any of the three systems were improved to give better cooling. Losses 
caused by insufficient debuncher cooling are a dominant limitation to stacking performance (when the other systems are working optimally). 
Figure 4

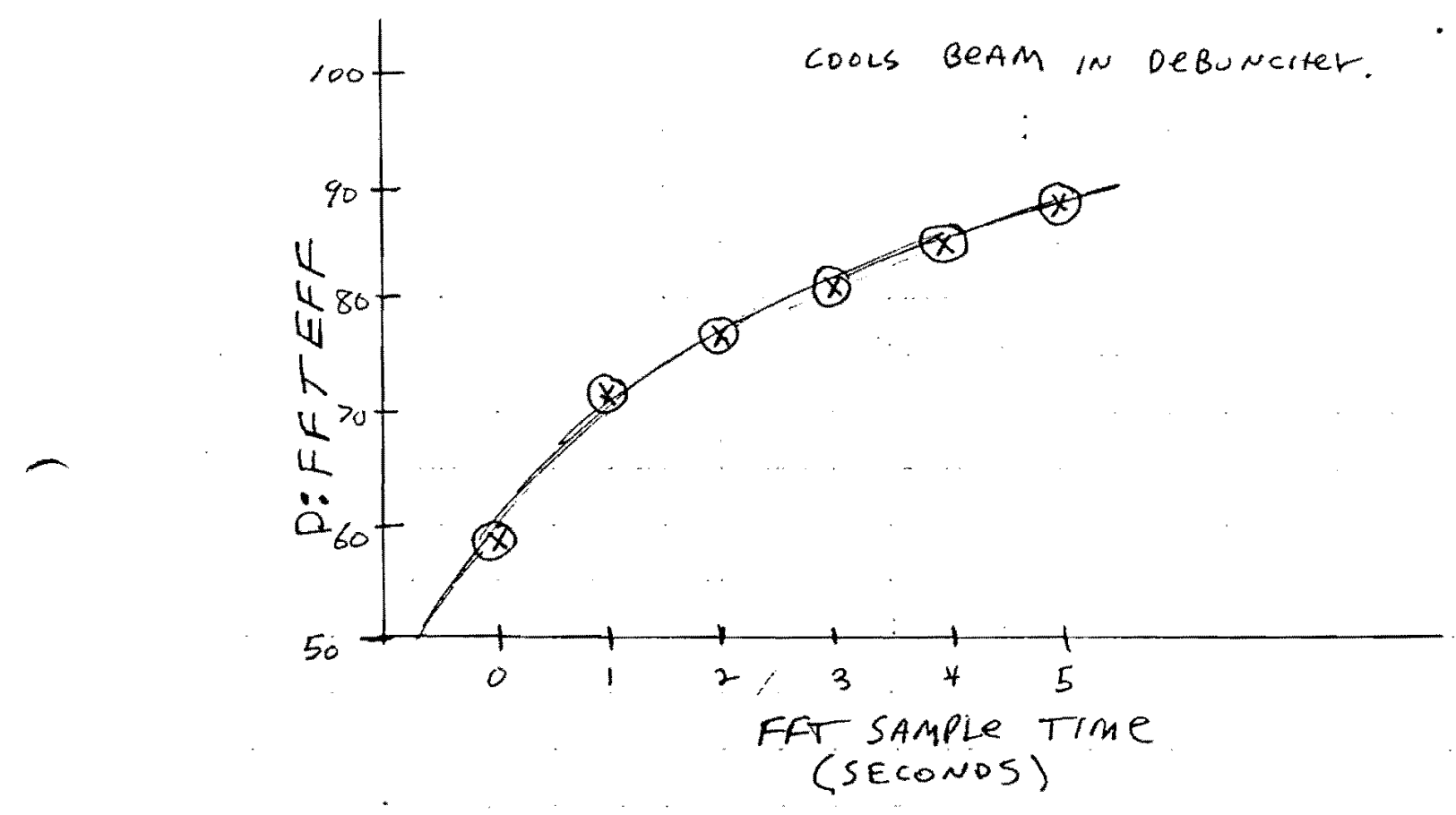


Figure 3

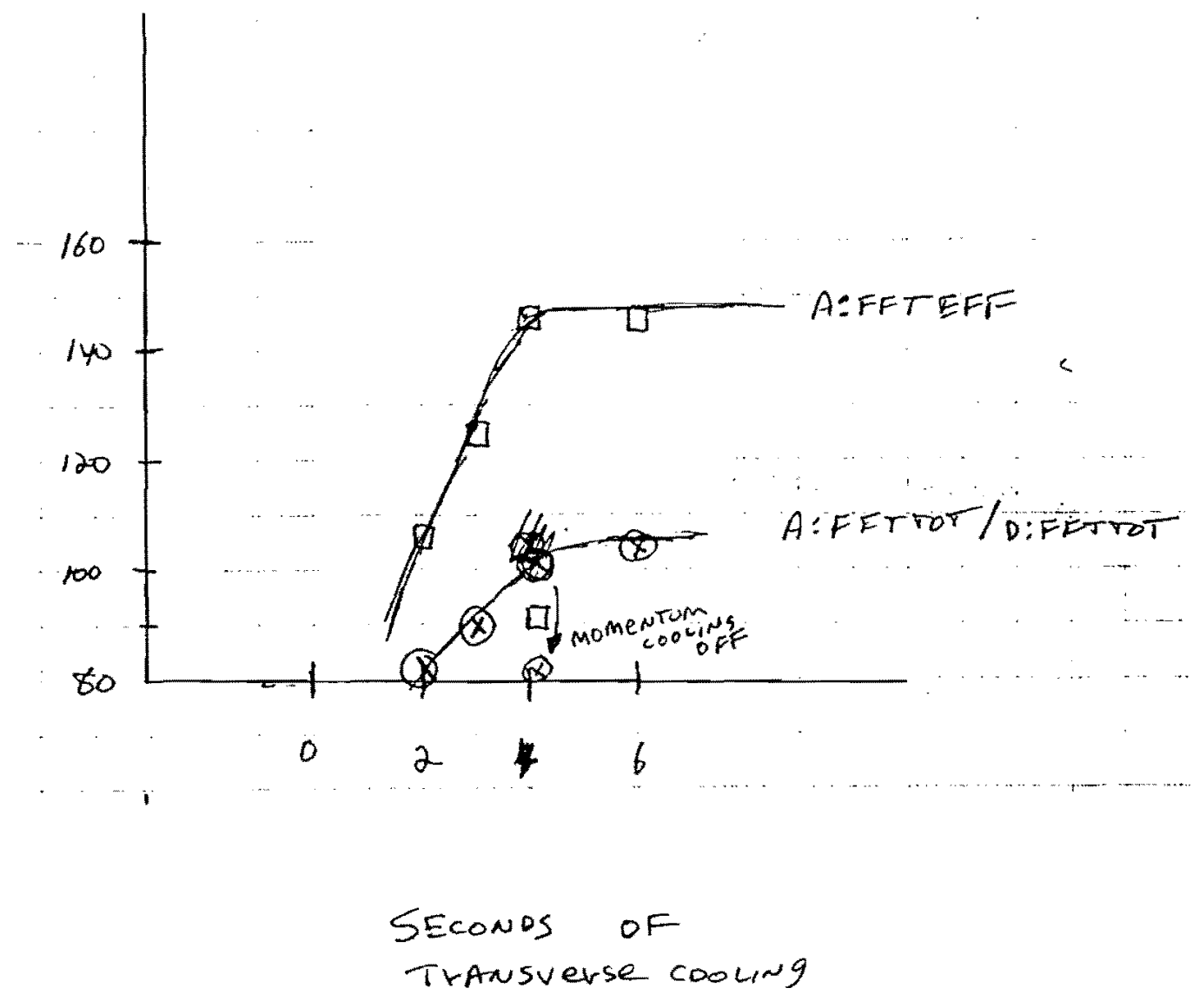




$$
\text { Figure } 4
$$

EFFICIENCY OF STACK TAIL SYSTEM

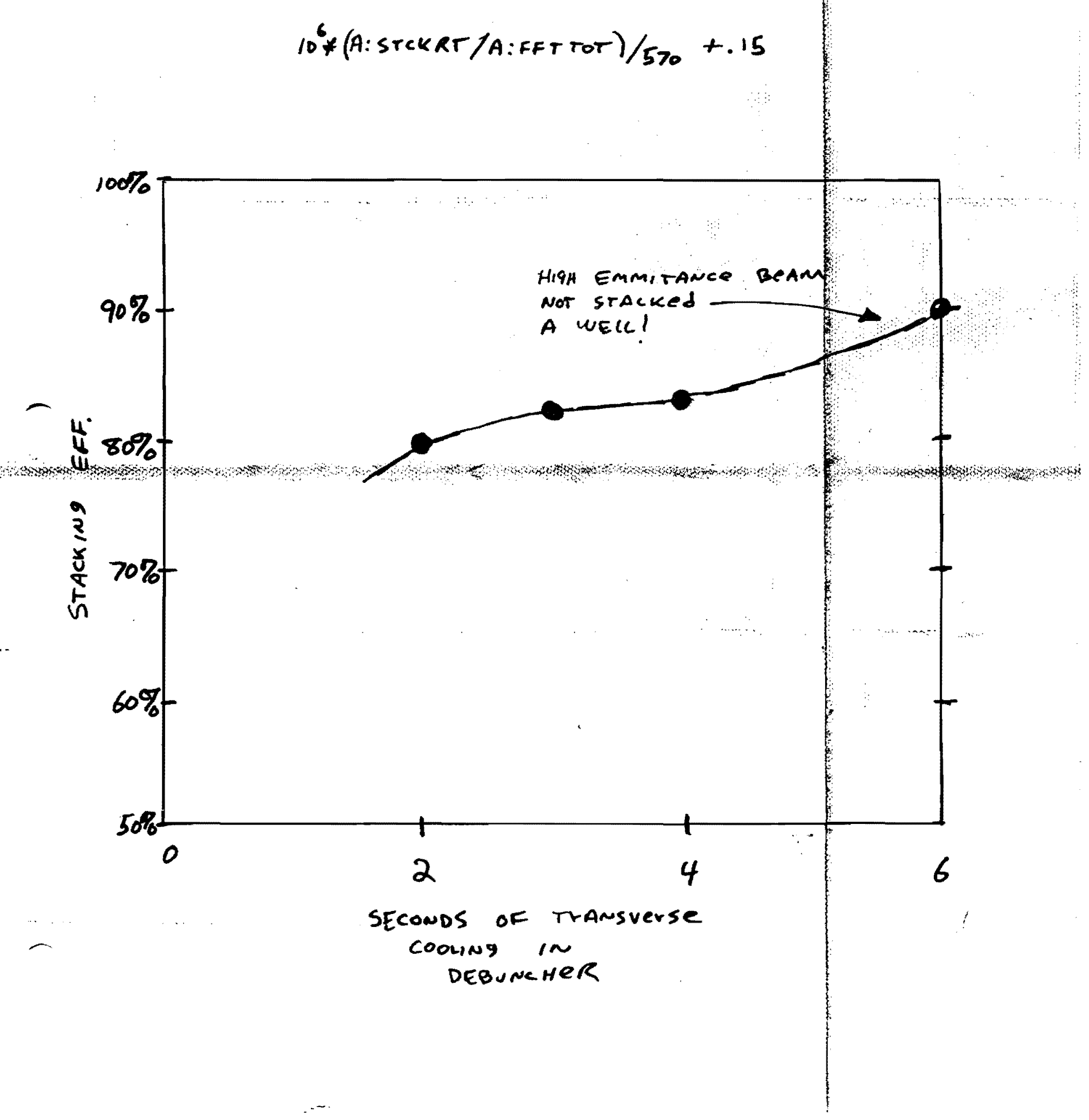




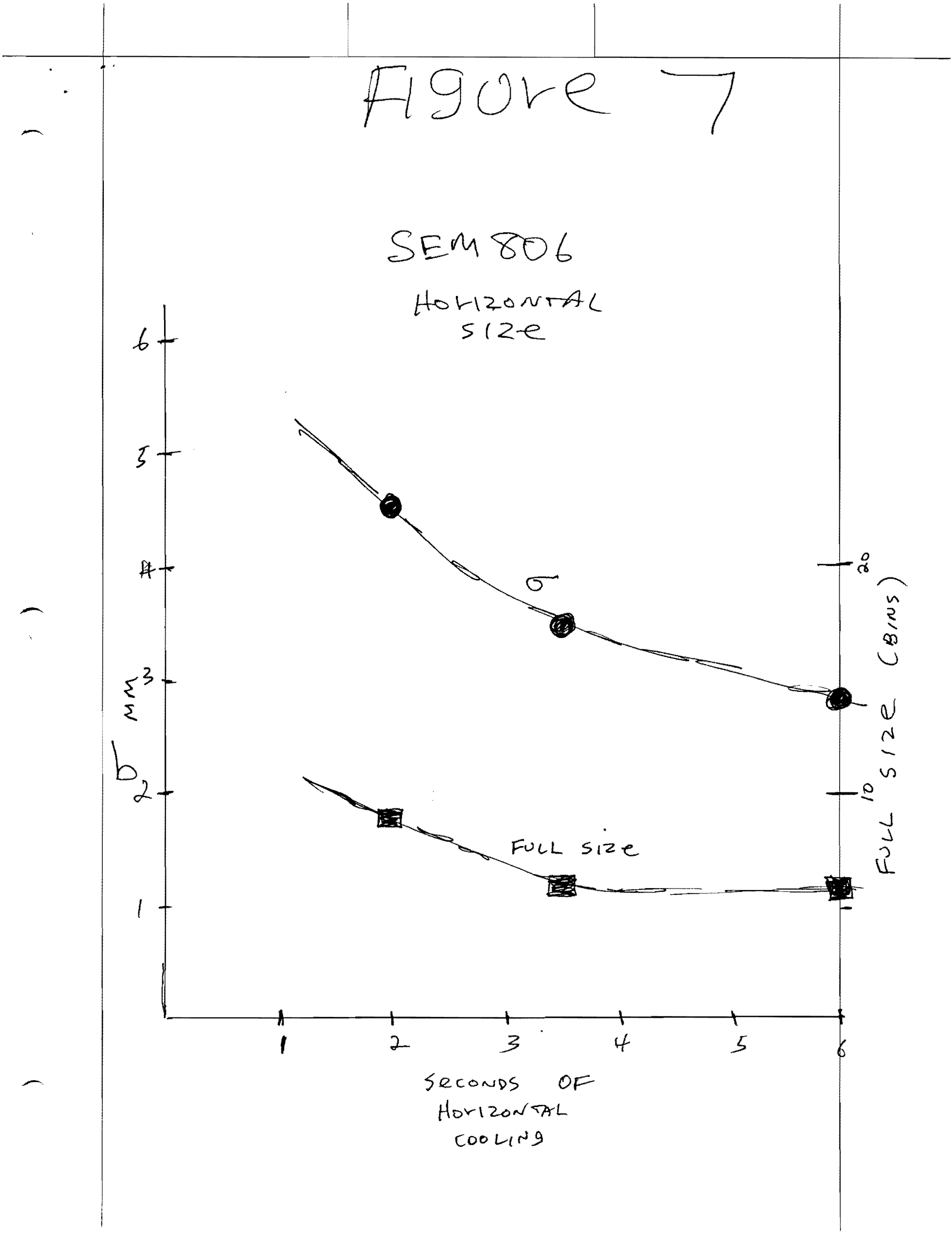




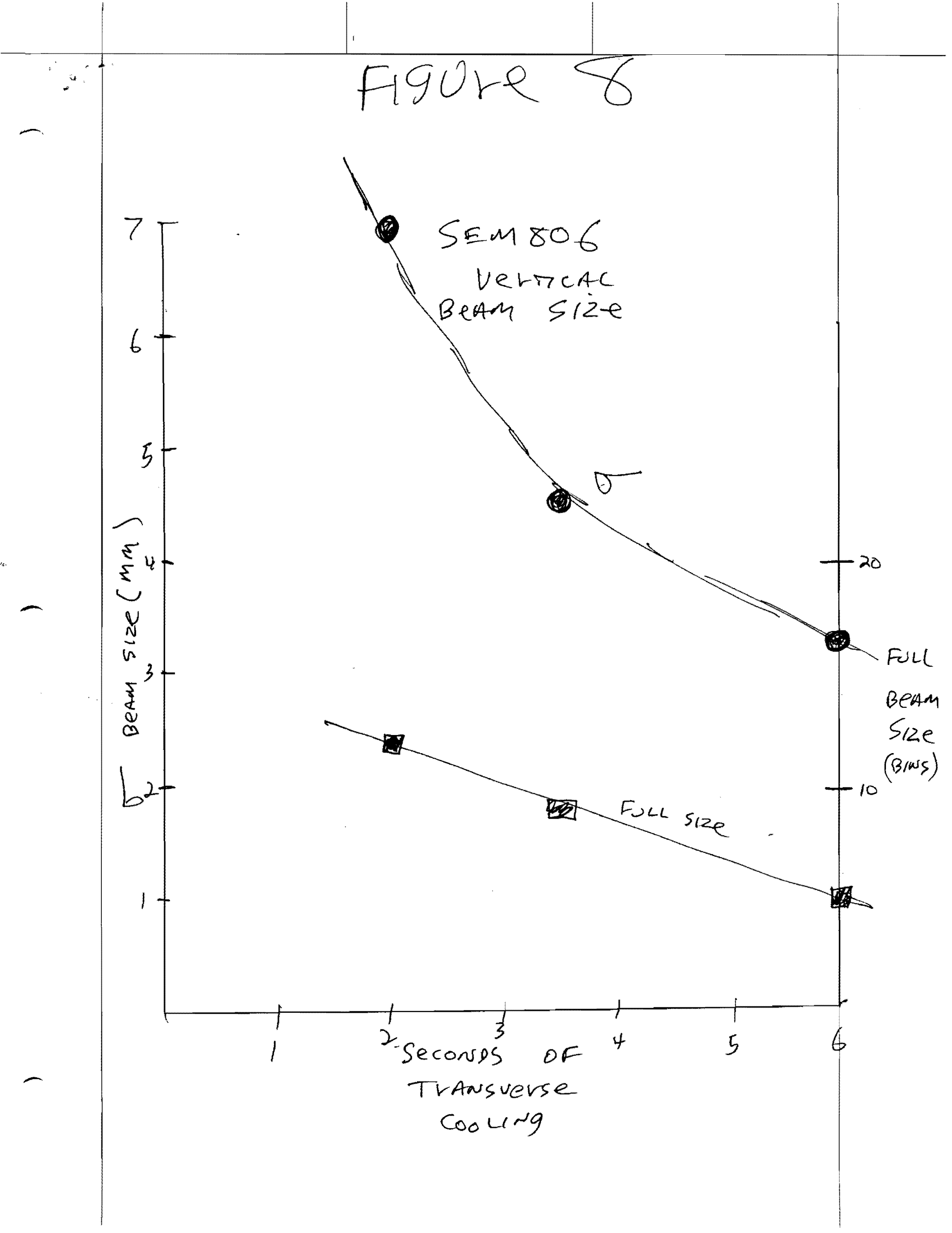

